\title{
Calling All Rheumatologists!
}

To the Editor:

These are difficult times. First responders are putting their lives at risk to save others. But this is our profession; we chose to work in the healthcare industry, knowing we would have daily exposure to different people with diverse ailments. However, nobody chose this profession thinking they would one day be part of a pandemic, fearing for their lives and those of their loved ones by proxy. Nobody in the United States thought that gloves and masks and ventilators could run short. But this is happening. It is happening now and it is happening fast.

Intensivists, emergency response teams, emergency room and primary care providers are on the front lines, identifying and treating these cases of the coronavirus disease 2019 (COVID-19; the disease caused by SARS-CoV-2). Everyone is on edge; it is only a matter of time before we are asked to step into the inpatient realm of the COVID wards. We douse our countertops, keyboards, and computer accessories with alcohol. We douse ourselves with sanitizer and soap. No one knows what else to do to stay safe.

The rheumatology community is getting more attention than it is used to, with an expedited US Food and Drug Administration approval for a drug we have been using for over 50 years - for something we say should be sprinkled in the water of all patients with systemic lupus erythematosus. What is considered a mild medication for rheumatologists bears toxic implications for other providers not accustomed to using it. And they are not wrong for it. Tocilizumab is being used in clinical trials and otherwise for use in COVID-19 cases believed to have cytokine release syndrome (CRS). Are our rheumatology colleagues chiming in? For a medication we have been using in rheumatoid arthritis and CRS [also called macrophage activation syndrome (MAS)], we should. Our contribution to this pandemic is to guide some of these biologic agents being studied in this deadly infection. Our knowledge of MAS cases will prove beneficial in specific COVID-19 cases. Do we need to enter the room of a patient with COVID-19 infection to weigh in? No. But we should be reviewing laboratory markers to identify CRS where there may be a window of opportunity to address the hyperimmune response to this infection.

We acknowledge the studies from years past using biologic agents during active viral infections, but it is now our duty to educate ourselves and others in the identification and management of CRS in the setting of an active COVID-19 infection. As Dr. Martin Luther King Jr. said, "The ultimate measure of a man is not where he stands in moments of comfort and convenience, but where he stands at times of challenge and controversy." So, to my colleagues, rheumatologists or otherwise, let us stand together in this challenging time and contribute in all ways possible. This, too, shall pass.

VANEET KAUR SANDHU, MD, FACR, RhMSUS, Division of Rheumatology, Department of Internal Medicine, Loma Linda University, Loma Linda,

California, USA. Address correspondence to Dr. V.K. Sandhu (c/o Cindy Burkett), 11234 Anderson St., MC 1516, Loma Linda, California 92354,

USA.E-mail: vksandhu@1lu.edu

First Release May 15 2020; J Rheumatol 2020;47:7;

doi:10.3899/jrheum.200421 